\title{
On a macroscopic limit of a kinetic model of alignment
}

\author{
Jacek Banasiak \\ School of Mathematical Sciences, University of KwaZulu-Natal \\ Durban, South Africa \\ Institute of Mathematics, Technical University of Łódź, \\ Łódź, Poland \\ banasiak@ukzn.ac.za \\ and \\ Mirosław Lachowicz \\ Institute of Applied Mathematics and Mechanics \\ Faculty of Mathematics, Informatics and Mechanics, University of Warsaw \\ ul. Banacha 2, 02-097 Warsaw, Poland \\ lachowic@mimuw . edu . pl
}

\begin{abstract}
In the present paper the macroscopic limits of the kinetic model for interacting entities (individuals, organisms, cells) proposed in [3] are studied. The kinetic model is one-dimensional and entities are characterized by their position and orientation (+/-) with swarming interaction controlled by the sensitivity parameter $\gamma$. The macroscopic limits of the model are considered for solutions close either to the diffusive (isotropic) or to the aligned (swarming) equilibrium states for various $\gamma$. In the former case the classical linear diffusion equation results whereas in the latter a traveling wave solution does both in the zeroth ('Euler') and first ('Navier-Stokes') order of approximation.
\end{abstract}

\section{Introduction}

The paper [3] focused on biological alignment (swarming) characterized as coherent motion of groups of entities (individuals, organisms or cells) into the same direction. We understand alignment as an adaptation of orientation to that of neighborhood. It results from the interplay of the behaviors of entities by local interactions. Swarms, also called herds, flocks, schools, clusters depending on whether they refer, respectively, to insects, mammals, birds, fish, bacteria and cells are often observed in nature. The typical examples are herds of sheep, flocks of birds or schools of fish (see References in [3, and 11, 19, 29, 30). In some of these phenomena alignment is a typical behavior. 
The phenomena of alignment (swarming), viewed as a cooperative phenomenon arising from the interaction of a number of entities, can be analyzed by mathematical models. The entities are characterized by their position, orientation (velocity) and migrate in space and time. Various microscopic, mesoscopic and macroscopic models describing swarming have been proposed. The dynamics of alignment in the space homogeneous case was considered e.g. in [12, 21. Microscopic models, for example the cellular automaton approach, 4, 11, and a simulation model by Ben-Jacob et al. [5] allows to distinguish individual entities. By means of a macroscopic model based e.g. on hydrodynamics (see 28), it is possible to study the dynamics of densities of entities. The review of various individual-based models based on ODEs is presented in 8. In a number of papers the authors have studied stochastic models for swarm aggregation, where the individuals, in addition to the classical Brownian random dispersal, are subject to long range attraction and short range repulsion. For instance, see [6] and references therein, under suitable laws of large numbers it was shown that, for a large number of individuals, the evolution of the empirical distribution of the population can be approximated using a suitable limit nonlinear degenerate and nonlocal parabolic equation. In [14] a class of stochastic individual-based models, written in terms of coupled velocity jump processes, was presented and analyzed. Moreover, in the limit of large populations, a system of two kinetic equations with nonlocal and nonlinear right hand side was derived and analyzed.

Mesoscopic models have been considered e.g. in [18, 3, 24, 9, 1]; see references therein. Such models are usually of Boltzmann type, i.e., they are related to a statistical description of one test entity.

The relations between the microscopic, mesoscopic and macroscopic models is one of the most challenging mathematical problem. Its importance is particularly visible in justification of the macroscopic models by a well-defined behavior of the entities of the system - see e.g. [6, 7, 8, 10, 14, 15, 16, 20, 22. and references therein.

In the present paper we study the macroscopic limits for the discrete velocity Boltzmann-type (mesoscopic) model based on a well-defined microscopic 'majority-choice' interaction introduced in [3. We are interested in the formation of alignment of the population of entities that do not possess a leader but polarization arises as a result of local alignment interactions.

The model, proposed in [3], is one-dimensional and the entities are characterized by their position and orientation (+/-) while the alignment (swarming) interaction is characterized by a sensitivity parameter $\gamma$. In the present paper the macroscopic limits of the model are considered for solutions either close to the diffusive (isotropic), or to the aligned (swarming), equilibrium states for various values of $\gamma$. In the former case, the classical linear diffusion equation results whereas in the latter we obtain a traveling wave solution both in the zeroth ('Euler') and the first ('Navier-Stokes') order of approximation. An interesting feature of the considered model is that the nonlinear kinetic model in the 'hydrodynamic' limits gives, in both cases, linear macroscopic equations. Moreover, it follows, at least formally, that all terms of the asymptotic expan- 
sion of the bulk part of the approximation, which are complementary to the traveling wave solution (its' 'kinetic' part), vanish and thus the bulk part of the approximation only consists of the traveling wave, whereas the complementary part is made up only of the initial layer terms.

The plan of the paper is as follows. Section 2 presents the model that was introduced in [3]. In Section [3 we prove a unified existence and uniqueness result valid both in weak and strong interaction cases. Section 4 deals with formal macroscopic limits of the model. This section also indicates some interesting open problems: the hydrodynamic limit in the 'diffusive' case. Finally we propose an asymptotic result in the 'aligned' case. The result is proved in the spirit of the Tikhonov-Vasileva theory (cf. [27]) that was formulated only in the finite-dimensional dynamical system case. Therefore it is an example of a generalization of the theory available for ODEs into some infinite dimensional case.

\section{The model}

Let $f=f(t, j, x)$ be the probability that an individual is at time $t$ at point $x$ with orientation $j$, where $t>0, x \in \mathbb{R}$ and $j \in\{-1,1\}$. Here the orientation plays the role of discrete velocity in the sense that it give the direction of motion.

We describe the migration together with the changes of the orientation variable where the latter is modeled by an interaction operator. We have the following general expression (valid for every migration-interaction case):

$$
f(t+\Delta t, j, x+j \Delta x)=f(t,-j, x) \mathfrak{P}(t,-j, x)+f(t, j, x) \mathfrak{P}^{\prime}(t, j, x),
$$

where

$$
\mathfrak{P}(t, j, x)=\text { Probability (a change of orientation in } \Delta t \mid(t, j, x)),
$$

is the probability of a change of orientation in the interval of time $[t, t+\Delta t]$ of an individual that is at $t$ at position $x$ with orientation $j$ and

$$
\begin{aligned}
& \mathfrak{P}^{\prime}(t, j, x)= \\
& \text { Probability }(\text { no changes of orientation in } \Delta t \mid(t, j, x))= \\
& 1-\mathfrak{P}(t, j, x) .
\end{aligned}
$$

Different choices of the probabilities $\mathfrak{P}$ give rise to different models, see e.g. [3] where, in particular, we proposed

$$
\mathfrak{P}(t, j, x)=\frac{\chi\left(\sum_{k, l= \pm 1} f(t, k, x+a l)>0\right)\left(\sum_{l= \pm 1} f(t,-j, x+a l)\right)^{\gamma}}{\left(\sum_{l= \pm 1} f(t,-j, x+a l)\right)^{\gamma}+\left(\sum_{l= \pm} f(t, j, x+a l)\right)^{\gamma}} \Delta t
$$


where $a>0$ and $\gamma>0$ are parameters and $\sum_{l= \pm 1} f(t, j, x+a l)$ is the neighborhood density in direction $j, \chi($ true $)=1, \chi($ false $)=0$. The parameter $\gamma$ describes sensitivity of the interaction. If $\gamma$ is small (close to 0 ), then the probability of a change of orientation only weakly depends on the actual orientation. On the other hand, for large $\gamma$ the probability of a change of orientation strongly depends on the actual orientation.

Assuming that $a=\Delta x$ and $\Delta x=j \Delta t$, in the limit $\Delta t \rightarrow 0$, we obtain the following system of two equations

$$
\begin{aligned}
& \partial_{t} f(t, j, x)+j \partial_{x} f(t, j, x)=\frac{\chi\left(\sum_{k= \pm 1} f(t, k, x)>0\right)}{(f(t,-j, x))^{\gamma}+(f(t, j, x))^{\gamma}} \\
& \times\left(f(t,-j, x)(f(t, j, x))^{\gamma}-f(t, j, x)(f(t,-j, x))^{\gamma}\right), \quad j=-1,1 .
\end{aligned}
$$

For $\gamma=1$, Eq. (3) decouples into two free-streaming equations. Throughout the paper we consider either $\gamma \in] 0,1[$ or $\gamma>1$.

The non-negative and non-zero equilibrium solutions $\bar{f}$, corresponding to the space homogeneous version of Eq. (3), can be found from

$$
\bar{f}_{-1}\left(\bar{f}_{1}\right)^{\gamma}-\bar{f}_{1}\left(\bar{f}_{-1}\right)^{\gamma}=0 .
$$

The only non-zero solutions $\bar{f}=\left(\bar{f}_{1}, \bar{f}_{-1}\right)$ of (4) are given by

$$
\bar{f}_{1}=\bar{f}_{-1}>0,
$$

or

$$
\bar{f}_{j}=0 \quad \text { and } \bar{f}_{-j}>0, \quad \text { for some } j \in\{-1,1\} .
$$

Equation (5) corresponds to equal probabilities of both orientations (a diffusive picture), whereas Eq. (6) is related to an aligned picture.

In the space-homogeneous case the trajectories of the corresponding ODEs are contained in the straight lines defined by

$$
f_{-1}+f_{1}=c, \quad \text { where } \quad c=f_{-1}(0)+f_{1}(0)>0,
$$

and have different types of behavior according to the value of $\gamma$. For $\gamma>1$

$$
\begin{aligned}
& \lim _{t \rightarrow \infty} f_{-1}=0, \quad \lim _{t \rightarrow \infty} f_{1}=f_{1}(0)+f_{-1}(0), \quad \text { for } f_{1}(0)>f_{-1}(0), \\
& \lim _{t \rightarrow \infty} f_{-1}=f_{1}(0)+f_{-1}(0), \quad \lim _{t \rightarrow \infty} f_{1}=0, \quad \text { for } f_{1}(0)<f_{-1}(0),
\end{aligned}
$$

whereas for $0<\gamma<1$ we have

$$
\lim _{t \rightarrow \infty} f_{1}=\lim _{t \rightarrow \infty} f_{-1}=\frac{f_{1}(0)+f_{-1}(0)}{2} .
$$


The important problem whether the similar behavior can be observed in the spatially nonhomogeneous case remains open.

We note that the spatially nonhomogeneous Eq. (3) admits simple solutions

$$
f(t, j, x)=f(t,-j, x)=\text { const. } \geq 0,
$$

and

$$
f(t, j, x)=0, \quad f(t,-j, x)=\phi(x+j t), \quad j=1,-1,
$$

where $\phi$ is a given non-negative function, obtained by equating both sides of (31) to zero.

Thus we observe that the possible asymptotic behavior for Eq. (3) may differ from that of the Carleman-type equations studied in [23, 13, 25, 26].

\section{Existence}

The (global) existence and uniqueness results for $\gamma>1$ as well as the existence of an entropy functional were established in [3] taking advantage of the Lipschitz continuity and the conservativeness of the system.

Here we propose the global existence and uniqueness results in an appropriate Banach space for both $\gamma>1$ and $\gamma<1$. We will consider one dimensional periodic boundary conditions, that is, we assume $x \in \mathbb{T}$, but similar results are possible for $x \in \mathbb{T}^{d}$, where $d \geq 1$ and $\mathbb{T}^{d}$ is a $d$-dimensional torus.

Let $X_{1}$ be the space of real-valued functions equipped with the norm

$$
\|f\|_{1}=\sum_{j= \pm 1} \int_{\mathbb{T}}|f(j, x)| \mathrm{d} x .
$$

The cone of non-negative functions in $X_{1}$ is denoted by $X_{1}^{+}$. We define the following operators

$$
Q[f]=Q^{+}[f]-Q^{-}[f],
$$

where

$$
Q^{+}[f](j, x)=\chi\left(\sum_{k= \pm 1} f(k, x)>0\right) \frac{f^{\gamma}(j, x) f(-j, x)}{f^{\gamma}(j, x)+f^{\gamma}(-j, x)}
$$

and

$$
Q^{-}[f](j, x)=\chi\left(\sum_{k= \pm 1} f(k, x)>0\right) \frac{f(j, x) f^{\gamma}(-j, x)}{f^{\gamma}(j, x)+f^{\gamma}(-j, x)} .
$$

Note that, for $f>0$,

$$
Q[f]=R[f]-f,
$$

where

$$
R[f](j, x)=f^{\gamma}(j, x) \frac{f(1, x)+f(-1, x)}{f^{\gamma}(1, x)+f^{\gamma}(-1, x)} .
$$

We denote

$$
\mathbb{Q}(h, g)(j, x)=\frac{h^{\gamma}(j, x) h(-j, x)}{h^{\gamma}(j, x)+g^{\gamma}(-j, x)},
$$


and

$$
\mathbb{P}(h, g)(j, x)=\frac{h^{\gamma}(-j, x)}{g^{\gamma}(j, x)+h^{\gamma}(-j, x)} .
$$

Let

$$
f^{\sharp}(t, j, x)=f(t, j, x+j t) .
$$

We define the following sequences $\left\{g_{n}\right\}_{n \in \mathbb{N}}$ and $\left\{h_{n}\right\}_{n \in \mathbb{N}}$ :

$$
g_{0} \equiv 0,
$$

while $h_{0}$ is the solution of the problem

$$
\dot{h}_{0}^{\sharp}(t, j, x)=h_{0}^{\sharp}(t,-j, x), \quad j=1,-1,\left.\quad h_{0}\right|_{t=0}=f_{0},
$$

where $f_{0} \in X_{1}^{+}$is the initial datum.

Moreover,

$$
\begin{aligned}
\dot{g}_{1}^{\sharp}+g_{1}^{\sharp} & =0,\left.\quad g_{1}\right|_{t=0}=f_{0}, \\
\dot{g}_{n}^{\sharp}+\mathbb{P}^{\sharp}\left(h_{n-1}, g_{n-1}\right) g_{n}^{\sharp} & =\mathbb{Q}^{\sharp}\left(g_{n-1}, h_{n-1}\right),\left.\quad g_{n}\right|_{t=0}=f_{0},
\end{aligned}
$$

for $n \geq 2$, and

$$
\dot{h}_{n}^{\sharp}+\mathbb{P}^{\sharp}\left(g_{n-1}, h_{n-1}\right) h_{n}^{\sharp}=\mathbb{Q}^{\sharp}\left(h_{n-1}, g_{n-1}\right),\left.\quad h_{n}\right|_{t=0}=f_{0},
$$

for $n \geq 1$.

Therefore we have

$$
\begin{gathered}
g_{1}(t, j, x)=f_{0}(x-j t) e^{-t} \\
\left\|h_{0}(t)\right\|_{1} \leq\left\|f_{0}\right\|_{1} e^{t},
\end{gathered}
$$

and

$$
0 \leq g_{1}(t, j, x) \leq f_{0}(x-j t) \leq h_{1}(t, j, x) \leq h_{0}(t, j, x) .
$$

Assuming that

$$
f_{0}(x) \geq \mu \quad \text { a.a. } x \in \mathbb{T}
$$

where $\mu$ is a positive $(>0)$ number, we obtain that on any compact interval $[0, T]$

$$
0<\mu e^{-T} \leq g_{1} \leq g_{2} \leq g_{3} \leq \ldots \leq h_{2} \leq h_{1} \leq h_{0} .
$$

Therefore, by the theorem of Beppo-Levy both sequences converge in $X_{1}$

$$
g_{n} \uparrow g, \quad h_{n} \downarrow h,
$$

in $X_{1}$ and

$$
g \leq h .
$$

On any fixed time interval $[0, T]$ we may apply the inequality

$$
0 \leq h^{\gamma}-g^{\gamma} \leq \gamma\left(\mu e^{-T}\right)^{\gamma-1}(h-g)
$$

to show that $g=h$. 
Thus $f:=g=h$ is a mild solution to the Eq. (3) on $[0, T], T>0$, in the following sense

$$
\begin{gathered}
f \in L^{\infty}\left(0, T ; X_{1}\right), \\
f^{\sharp}(t)+\int_{0}^{t} P^{\sharp}(f, f) f_{n}^{\sharp}=f_{0}+\int_{0}^{t} Q^{\sharp}(f, f) .
\end{gathered}
$$

for a.a. $t \in[0, T]$. The similar argument as the one used for $g=h$ shows that the solution is unique. Moreover, the solution is in $X_{1}^{+}$.

Therefore we have

Theorem 3.1. Let $f_{0} \in X_{1}^{+}$be such that (15) is satisfied for some positive $\mu$. Then, for any given $T>0$ there exists a unique mild solution $f=f(t) \in X_{1}$ of $E q$. (3) with the initial datum $f_{0}$ on $[0, T]$. Moreover,

- $f(t) \in X_{1}^{+}$for a.a. $t \in[0, T]$,

- $\mu e^{-T} \leq f(t)$ for a.a. $t \in[0, T]$,

- $\|f(t)\|_{1} \leq\left\|f_{0}\right\|_{1} e^{T}$ for a.a. $t \in[0, T]$.

Remark 3.2. Note that if (15) is satisfied, then $f$ is a mild solution of Eq. (3) in which each term is on the right hand side is separated from zero and thus Frèchet differentiable. Hence, the solution becomes a classical solution provided the initial datum is differentiable.

\section{Formal macroscopic analysis}

In this section we follow the strategy developed in the case of the so-called hydrodynamic limits for kinetic equations in [15].

It is well known that the macroscopic limit is defined by $\varepsilon \rightarrow 0$, where $\varepsilon>0$ is a suitable small parameter (the Knudsen number). Classically, see e.g. [15], Equation (3) can be considered in the following two dimensionless forms

$$
\left(\partial_{t}+j \partial_{x}\right) f(t, j, x)=\frac{1}{\varepsilon} Q[f](t, j, x),
$$

and

$$
\left(\varepsilon \partial_{t}+j \partial_{x}\right) f(t, j, x)=\frac{1}{\varepsilon} Q[f](t, j, x),
$$

where the nonlinear operator $Q$ is given by the RHS of Eq. (3). Equations (16) and (17) with the initial data

$$
\left.f\right|_{t=0}=F
$$

lead to singularly perturbed problems in the limit $\varepsilon \rightarrow 0$. 
We introduce some basic notation. The 0 -th order term in the Hilbert expansion is the function $M$ (a Maxwellian) which satisfies

$$
Q(M)=0 .
$$

Therefore

$$
M(t, j, x)=\eta_{j} \varrho(t, x), \quad \eta_{1}+\eta_{-1}=1
$$

where either

$$
\eta_{1}=\eta_{-1}=\frac{1}{2}
$$

or

$$
\eta_{k}=1, \quad \eta_{-k}=0
$$

for some $k \in\{-1,1\}$, and $\varrho$ is the macroscopic density. Therefore we conclude that two macroscopic limits can be considered for initial data close to (20) either with (21) or (22). This is, however, strictly related to stability properties of the solutions.

Consider the first Fréchet derivative (linearization) $L$ of the operator $Q$ at $M$. It has the form

$$
L f(j)=\frac{1-\gamma}{2}(f(-j)-f(j))
$$

if (21) holds, and

$$
L f(j)=\left\{\begin{array}{cl}
f(-k) & \text { for } j=k \\
-f(-k) & \text { for } j=-k
\end{array}\right.
$$

if (22) is satisfied. For simplicity the dependence of $t$ and $x$ is not explicitly indicated.

The null-space of $L$, called the hydrodynamic space, is spanned by the Maxwellian $M$. Since the aim of asymptotic analysis is to isolate the slow dynamics in the hydrodynamic space from fast, transient, behavior in the complementary space, the best way is split the phase space into subspaces reducing $L$. Hence, we call the kinetic space the space spanned by all remaining eigenvectors of $L$ (corresponding to eigenvalues with negative real parts). The kinetic space will be denoted by $W$. This can be done by spectral projections. For a general matrix $A$ having 0 as the dominant simple eigenvalue with the corresponding eigenvector with $e$ and $e^{*}$ being the eigenvector of the transpose $A^{*}$ corresponding to 0 and normalized so as $e \cdot e^{*}=1$, the spectral projection $\mathcal{P}$ onto the null-space of $A$ is given by

$$
\mathcal{P} y=\left(e^{*} \cdot y\right) e
$$

Then clearly the complementary projection $\mathcal{P}^{\perp}$ is defined as $\mathcal{P}^{\perp}=I-\mathcal{P}$, where $I$ is the identity matrix. Specifying this theory to the case in hand, we see that in the diffusive case (23) the null spaces of $L$ and $L^{*}=L$ are spanned by, respectively, $(1 / 2,1 / 2)$ and $(1,1)$ (where we have taken into account the normalization). Thus, in this case we have

$$
\mathcal{P}(f(1), f(-1))=\left(\frac{f(1)+f(-1)}{2}, \frac{f(1)+f(-1)}{2}\right)
$$


and

$$
\mathcal{P}^{\perp}(f(1), f(-1))=\left(\frac{f(1)-f(-1)}{2}, \frac{f(-1)-f(1)}{2}\right) .
$$

The situation is, however, different in both cases described by (22). For $k=1$ the eigenvector of $L$ corresponding to 0 is given by $(1,0)$, whereas $(0,1)$ is the respective eigenvector for $k=-1$. For the transpose matrix the eigenvector is given by $(1,1)$ in either case. Thus, we obtain

$$
\mathcal{P}(f(1), f(-1))=(f(1)+f(-1), 0)
$$

and

$$
\mathcal{P}^{\perp}(f(1), f(-1))=(-f(-1), f(-1)),
$$

if $k=1$, and

$$
\mathcal{P}(f(1), f(-1))=(0, f(1)+f(-1))
$$

with

$$
\mathcal{P}^{\perp}(f(1), f(-1))=(f(1),-f(1))
$$

for $k=-1$. We observe that the above expression give a preliminary justification for the terms diffusive and aligned picture. Indeed, in the first case the total density $\varrho=f(1)+f(-1)$ is uniformly spread between the two available directions of motion whereas in the other two the total density is concentrated along one of the directions of motion. Let us introduce an operator $D$ by the formula

$$
D(f(1, x), f(-1, x))=\left(\partial_{t} f(1, x)+\partial_{x} f(1, x), \partial_{t} f(-1, x)-\partial_{x} f(-1, x)\right) .
$$

Referring to the problem (16) we note (see [15]) that the abstract zeroth order ('Euler') macroscopic approximation is given by

$$
\mathcal{P} D M=0,
$$

whereas the first ('Navier-Stokes') order macroscopic approximation is given by

$$
\mathcal{P} D M=-\varepsilon \mathcal{P} D \mathcal{P}^{\perp} L^{-1} \mathcal{P}^{\perp} D M
$$

in terms of the macroscopic density $\varrho$.

Specifying for our three cases, we obtain the following formulae

$$
D M= \begin{cases}\frac{1}{2}\left(\partial_{t} \varrho+\partial_{x} \varrho, \partial_{t} \varrho-\partial_{x} \varrho\right) & \text { in the diffusive case } \\
\left(\partial_{t} \varrho+\partial_{x} \varrho, 0\right) & \text { in the aligned case, } \mathrm{k}=1 \\
\left(0, \partial_{t} \varrho-\partial_{x} \varrho\right) & \text { in the aligned case, } \mathrm{k}=-1,\end{cases}
$$

which, upon application of an appropriate $\mathcal{P}$, yield the following Euler approximating equations for the diffusive and aligned regimes with $k=1,-1$, respectively,

$$
\begin{array}{ll}
\partial_{t} \varrho & =0, \\
\partial_{t} \varrho+\partial_{x} \varrho & =0, \\
\partial_{t} \varrho-\partial_{x} \varrho & =0 .
\end{array}
$$


To derive the Navier-Stokes picture, first we find

$$
\mathcal{P}^{\perp} D M= \begin{cases}\left(\partial_{x} \varrho,-\partial_{x} \varrho\right) & \text { in the diffusive case, } \\
(0,0) & \text { in the aligned case, } \mathrm{k}=1, \\
(0,0) & \text { in the aligned case, } \mathrm{k}=-1,\end{cases}
$$

Clearly, the operator $L$ is not invertible but becomes such if reduced to the kinetic space $W$ which, in the diffusive case, is spanned by $(1,-1)$. We see that $\mathcal{P}^{\perp} D M \in W$ and the solution to $L(y, z)=\left(\partial_{x} \varrho,-\partial_{x} \varrho\right)$ subject to $y=-z$ is $\left(-\partial_{x} \varrho, \partial_{x} \varrho\right) /(1-\gamma)$. Next,

$$
D \mathcal{P}^{\perp} L^{-1} \mathcal{P}^{\perp} D M=(1-\gamma)^{-1}\left(-\partial_{t} \partial_{x} \varrho-\partial_{x}^{2} \varrho, \partial_{t} \partial_{x} \varrho-\partial_{x}^{2} \varrho\right)
$$

and finally

$$
\mathcal{P} D \mathcal{P}^{\perp} L^{-1} \mathcal{P}^{\perp} D M=-(1-\gamma)^{-1}\left(\partial_{x}^{2} \varrho, \partial_{x}^{2} \varrho\right)
$$

which, combined with $\mathcal{P} D M=\left(\partial_{t} \varrho, \partial_{t} \varrho\right)$, yields the first order macroscopic approximation (32) in the form

$$
\partial_{t} \varrho=\frac{\varepsilon}{1-\gamma} \partial_{x}^{2} \varrho
$$

Clearly, the Navier-Stokes approximation in either aligned case is again the respective Euler approximation.

Next we consider the diffusive picture regime for problem the parabolic scaling (17). Performing similar considerations we see that the zeroth order macroscopic approximation is given by

$$
\partial_{t} \varrho=\frac{1}{1-\gamma} \partial_{x}^{2} \varrho .
$$

Equations (34) and (35) are linear diffusion equations for $0<\gamma<1$, with the diffusion coefficients $\frac{\varepsilon}{1-\gamma}$ and $\frac{1}{1-\gamma}$, respectively. Linear dependence on $\varepsilon$ of the diffusion coefficient in the limit equation (34) in the case of scaling (16) is typical (cf. 15, 20). In the case of $\gamma>1$ Eqs. (34) and (35) are the (linear) backward diffusion equations. The backward diffusion equation is less mathematically tractable than the diffusion equation - see however [17, Example 8.6].

Thus, we cannot expect any stability properties in the diffusive regime if $\gamma>1$. On the other hand, one may expect that for $0<\gamma<1$ the asymptotic relationships between the solutions of (16) or (17) and (34) or (35), respectively, hold.

Next, let us consider the aligned regime for Eq. (16) with initial data (18). Let $k \in\{-1,1\}$ be fixed and, to avoid technical difficulties we consider only $\gamma>1$. We have seen that the macroscopic limit at both Euler and NavierStokes levels does not depend on the choice of $\gamma$ and is given by

$$
\partial_{t} \varrho+k \partial_{x} \varrho=0 .
$$

Equation (36) is a linear first order equation and for any smooth initial datum $F$ its unique solution is given by

$$
\varrho(t, x)=F(k, x-k t), \quad t \geq 0, \quad x \in \mathbb{T} .
$$


Clearly, the solution inherits all properties of $F$ such as boundedness, differentiability, etc. This solution is a traveling wave (in the direction $k$ ).

Next we investigate in more detail to aligned picture and, in particular, we provide a more refined expansion formulae. To simplify notation, let us fix $k=1$; the calculation for $k=-1$ being symmetric.

Then, following (27) and (28), we have the hydrodynamic part of the solution given by $\varrho=f_{1}+f_{-1}$ and the kinetic part defined as $w=f_{-1}$ so that

$$
\left(f_{1}, f_{-1}\right)=(1,0) \varrho+(-1,1) w .
$$

Using $f_{1}=\varrho-w$ and $f_{-1}=w$, we see that the system (16) can be written in hydrodynamic and kinetic variables as

$$
\begin{aligned}
\partial_{t} \varrho+\partial_{x} \varrho-2 \partial_{x} w & =0, \\
\partial_{t} w-\partial_{x} w & =\frac{1}{\varepsilon} \frac{(\varrho-w) w^{\gamma}-w(\varrho-w)^{\gamma}}{(\varrho-w)^{\gamma}+w^{\gamma}} .
\end{aligned}
$$

First we look for the bulk approximation.

We perform the asymptotic expansion in the spirit of the Chapman-Enskog, that is, we do not expand the hydrodynamic part and seek approximation $(\varrho, w)=\left(\varrho, w_{0}+\varepsilon w_{1}\right)$, see e.g. [20]. This produces

$$
\begin{aligned}
& \partial_{t} \varrho+\partial_{x} \varrho-2 \partial_{x}\left(w_{0}+\varepsilon w_{1}\right)=0, \\
& \varepsilon \partial_{t}\left(w_{0}+\varepsilon w_{1}\right)-\varepsilon \partial_{x}\left(w_{0}+\varepsilon w_{1}\right) \\
& \quad=\frac{\left(\varrho-\left(w_{0}+\varepsilon w_{1}\right)\right)\left(w_{0}+\varepsilon w_{1}\right)^{\gamma}-\left(w_{0}+\varepsilon w_{1}\right)\left(\varrho-\left(w_{0}+\varepsilon w_{1}\right)\right)^{\gamma}}{\left(\varrho-\left(w_{0}+\varepsilon w_{1}\right)\right)^{\gamma}+\left(w_{0}+\varepsilon w_{1}\right)^{\gamma}} .
\end{aligned}
$$

Taking $O(1)$ terms on both sides, we obtain

$$
0=\frac{\left(\varrho-w_{0}\right) w_{0}^{\gamma}-w_{0}\left(\varrho-w_{0}\right)^{\gamma}}{\left(\varrho-w_{0}\right)^{\gamma}+w_{0}^{\gamma}}
$$

which yields $w_{0}=0$ or $w_{0}=\varrho$. If we recognize that the kinetic part for $k=1$ is the hydrodynamic part for $k=-1$, then the latter option simply give the $k=-1$ case and we are left with $w_{0}=0$. To get $w_{1}$ we write

$$
\begin{aligned}
\frac{\left(\varrho-\varepsilon w_{1}\right)\left(\varepsilon w_{1}\right)^{\gamma}-\varepsilon w_{1}\left(\varrho-\varepsilon w_{1}\right)^{\gamma}}{\left(\varrho-\varepsilon w_{1}\right)^{\gamma}+\left(\varepsilon w_{1}\right)^{\gamma}} & =\frac{-\varepsilon \varrho^{\gamma} w_{1}+O\left(\varepsilon^{\min \{2, \gamma\}}\right)}{\varrho^{\gamma}\left(1-\varepsilon \gamma w_{1} \varrho^{-1}+O\left(\varepsilon^{\min \{2, \gamma\}}\right)\right)} \\
& =-\varepsilon w_{1}+O\left(\varepsilon^{\min \{2, \gamma\}}\right)
\end{aligned}
$$

and, since the remaining LHS terms in (40) are $O\left(\varepsilon^{2}\right)$, it follows that $w_{1}=0$. Hence, we recovered the relevant, second, equation from (33). We note that in this model and the presented level of expansion Chapman-Enskog and Hilbert methods yield the same approximation.

Remark 4.1. By induction we can prove that all terms of the expansion $w=$ $w_{0}+\varepsilon w_{1}+\ldots+\varepsilon^{n} w_{n}+\ldots$ are zero. Indeed, if $w_{k}=0$ for $k=0,1, \ldots, n-1$, 
the calculation as above gives

$$
\begin{aligned}
& \frac{\left(\varrho-\varepsilon^{n} w_{n}\right)\left(\varepsilon^{n} w_{n}\right)^{\gamma}-\varepsilon^{n} w_{n}\left(\varrho-\varepsilon^{n} w_{n}\right)^{\gamma}}{\left(\varrho-\varepsilon^{n} w_{n}\right)^{\gamma}+\left(\varepsilon^{n} w_{n}\right)^{\gamma}} \\
& \quad=\frac{-\varepsilon^{n} \varrho^{\gamma} w_{n}+O\left(\varepsilon^{\min \{2 n, \gamma n\}}\right)}{\varrho^{\gamma}\left(1-\varepsilon^{n} \gamma w_{n} \varrho^{-1}+O\left(\varepsilon^{\min \{2 n, \gamma n\}}\right)\right)}=-\varepsilon^{n} w_{n}+O\left(\varepsilon^{\max \{2 n, \gamma n\}}\right)
\end{aligned}
$$

and since the LHS of (40) now is $O\left(\varepsilon^{n+1}\right)$, we find $w_{n}=0$.

Next we incorporate the initial layer correction and look at the approximation $(\varrho(t), w(t)) \approx\left(\bar{\varrho}(t)+r\left(\frac{t}{\varepsilon}\right), h\left(\frac{t}{\varepsilon}\right)\right)$, where $\bar{\varrho}$ is the solution to the initial value problem

$$
\partial_{t} \bar{\varrho}+\partial_{x} \bar{\varrho}=0, \quad \bar{\varrho}(0, x)=\varrho_{0}(x)=F(1, x) .
$$

Then, denoting $\tau=\frac{t}{\varepsilon}$, the initial layer corrections $r, h$ are obtained by an asymptotic expansion of

$$
\begin{aligned}
\partial_{t} \bar{\varrho}+\varepsilon^{-1} \partial_{\tau} r+\partial_{x}(\bar{\varrho}+r)-2 \partial_{x} h & =0, \\
\partial_{\tau} h-\varepsilon \partial_{x} h & =\frac{(\varrho+r-h) h^{\gamma}-h(\varrho+r-h)^{\gamma}}{(\varrho+r-h)^{\gamma}+h^{\gamma}} .
\end{aligned}
$$

Since we expect $r$ to decay exponentially to 0 as $\tau \rightarrow \infty$, from the first equation we obtain $r=0$. Then, setting $\varepsilon=0$ in the second equation and taking into account that $\left.\varrho(t, x)\right|_{\varepsilon=0}=\left.\bar{\varrho}(\varepsilon \tau)\right|_{\varepsilon=0}=\varrho_{0}$ we obtain

$$
\begin{aligned}
\partial_{\tau} h & =\frac{\left(\varrho_{0}-h\right) h^{\gamma}-h\left(\varrho_{0}-h\right)^{\gamma}}{\left(\varrho_{0}-h\right)^{\gamma}+h^{\gamma}}, \\
h(0, x) & =F(-1, x) .
\end{aligned}
$$

\section{$5 \quad$ Aligned picture}

We consider the 'aligned picture' for Eq. (16) in the case $\gamma>1$. We assume that $k \in\{-1,1\}$ is fixed.

Let $x \in \mathbb{T}$, where $\mathbb{T}$ is the 1 -dimensional torus. All functions are then interpreted as periodic functions on the unit interval $[0,1]$.

Let $X_{\infty}$ be the Banach space of continuous functions defined on $\mathbb{T}$ with the norm

$$
\|f\|_{\infty}=\sum_{j \in\{-1,1\}} \sup _{x \in \mathbb{T}}|f(j, x)| .
$$

We consider Eq. (16), i.e.

$$
\varepsilon\left(\partial_{t} f_{j}+j \partial_{x} f_{j}\right)=\frac{\chi\left(f_{j}+f_{-j}>0\right)}{f_{j}^{\gamma}+f_{-j}^{\gamma}}\left(f_{-j} f_{j}^{\gamma}-f_{j} f_{-j}^{\gamma}\right), \quad j=k,-k,
$$

where $f_{j}(t, x)=f(t, j, x)$. 
Following the formal results derived in the previous section, we are looking for the solution to Eq. (45) in the following form

$$
f_{k}(t, x)=\bar{\varrho}(t, x)-h\left(\frac{t}{\varepsilon}, x\right)+\varepsilon u(t, x), \quad f_{-k}(t, x)=h\left(\frac{t}{\varepsilon}, x\right)+\varepsilon v(t, x),
$$

where $(\varepsilon u, \varepsilon v)$ is the error of the approximation. We recall that $\varrho=\varrho(t, x)$ is the 'bulk solution', $h=h(\tau, x)$ is the 'initial layer solution' term, $\tau=\frac{t}{\varepsilon}$ is the stretched time variable. Then the error satisfies

$$
\begin{aligned}
& \varepsilon^{2}\left(\partial_{t} u+k \partial_{x} u\right)-\partial_{\tau} h-\varepsilon k \partial_{x} h \\
& =\frac{\chi(\bar{\varrho}+\varepsilon(u+v)>0)\left((h+\varepsilon v)(\bar{\varrho}-h+\varepsilon u)^{\gamma}-(\bar{\varrho}-h+\varepsilon u)(h+\varepsilon v)^{\gamma}\right)}{(\bar{\varrho}-h+\varepsilon u)^{\gamma}+(h+\varepsilon v)^{\gamma}}, \\
& \varepsilon^{2}\left(\partial_{t} v-k \partial_{x} v\right)+\partial_{\tau} h-\varepsilon k \partial_{x} h \\
& =\frac{\chi(\varrho+\varepsilon(u+v)>0)\left((\bar{\varrho}-h+\varepsilon u)(h+\varepsilon v)^{\gamma}-(h+\varepsilon v)(\bar{\varrho}-h+\varepsilon u)^{\gamma}\right)}{(\bar{\varrho}-h+\varepsilon u)^{\gamma}+(h+\varepsilon v)^{\gamma}},
\end{aligned}
$$

where $\bar{\varrho}$ satisfies (37), $h=h(\tau, x)$ satisfies (43), (44) and $\varrho_{0}(x)=\varrho(0, x)=$ $F(k, x)$.

First we consider the initial layer problem for $h$. We have

Lemma 5.1. Let $k \in\{-1,1\}$ and the initial data $F \in X$ be such that

$$
F(-k, x) \geq \mu, \quad \forall x \in \mathbb{T},
$$

for some $\mu>0$. There exists $c_{\gamma}>1$ such that, if

$$
F(k, x)>c_{\gamma} F(-k, x) \quad \forall x \in \mathbb{T},
$$

then there exists a unique solution $h=h(\tau, x)$ to the problem (43), (44) for any $\tau>0$ and

$$
0<h(\tau, x) \leq F(-k, x) e^{-\delta \tau} \quad \forall \tau>0 \quad \forall x \in \mathbb{T},
$$

for some $\delta>0$. Moreover if, additionally, $F$ is continuously differentiable and $c_{\gamma}$ is sufficiently large, then the solution $h=h(\tau, x)$ to (43), (44) is continuously differentiable and

$$
\left|\partial_{x} h(\tau, x)\right| \leq \text { const. }\left(\|F\|_{\infty}+\left\|\partial_{x} F\right\|_{\infty}\right) e^{-\delta_{1} \tau} \quad \forall \tau>0 \quad \forall x \in \mathbb{T},
$$

for some $\delta_{1}>0$. 
Proof. Problem (43), (44) is an ODE problem, where the variable $x$ is a parameter. For $\gamma>1$ the operator defined by the RHS of (43) is Lipschitz continuous on $C(\mathbb{T})$. Under the condition (49) for a.a. $x \in \mathbb{T}$ and $\tau=0$ the RHS of Eq. (43) is negative. Therefore $h$ strictly decreases with respect to the variable $\tau$ for all $x \in \mathbb{T}$. Now,

$$
\begin{array}{r}
\frac{1}{\left(\varrho_{0}-h\right)^{\gamma}+h^{\gamma}}\left(\left(\varrho_{0}-h\right) h^{\gamma}-h\left(\varrho_{0}-h\right)^{\gamma}\right) \\
=-h \frac{\left(\varrho_{0}-h\right)\left(\left(\varrho_{0}-h\right)^{\gamma-1}-h^{\gamma-1}\right)}{\left(\varrho_{0}-h\right)^{\gamma}+h^{\gamma}} .
\end{array}
$$

Since, by (49),

$$
0 \leq \vartheta:=\frac{h}{\varrho_{0}-h} \leq \frac{F(-k)}{F(k)-F(-k)} \leq \frac{1}{c_{\gamma}-1}
$$

we can write

$$
\frac{\left(\varrho_{0}-h\right)\left(\left(\varrho_{0}-h\right)^{\gamma-1}-h^{\gamma-1}\right)}{\left(\varrho_{0}-h\right)^{\gamma}+h^{\gamma}}=\frac{1-\vartheta^{\gamma-1}}{1+\vartheta \gamma}:=\delta>0
$$

uniformly in $x \in[0,1]$ and $\tau>0$. We note that $\delta$ can be made as close to 1 as we wish by making $c_{\gamma}$ sufficiently large but since close to the equilibrium $h=0$ the left hand side of (52) is close to $1, \delta$ could not exceed 1 . This leads to the conclusion that $h$ decays exponentially to 0 for all $x \in \mathbb{T}$ and the rate of convergence can be controlled by $e^{-\delta \tau}$ uniformly in $x \in \mathbb{T}$. Moreover it is strictly positive. Thus the unique solution of Eq. (43) satisfies (50).

It is evident that if $F$ is continuously differentiable with respect to $x$, then also $h$ is. We denote $h^{\prime}=\partial_{x} h$ and $\varrho_{0}^{\prime}=\partial_{x} \varrho_{0}$. The function $h^{\prime}(\tau, x)$ satisfies

$$
\begin{aligned}
& \partial_{\tau} h^{\prime}=\frac{\gamma\left(\varrho_{0}-h\right)^{\gamma-1}\left(\varrho_{0}^{\prime}-h^{\prime}\right)+\gamma h^{\gamma-1} h^{\prime}}{\left(\left(\varrho_{0}-h\right)^{\gamma}+h^{\gamma}\right)^{2}}\left(\varrho_{0}-h\right) h\left(\left(\varrho_{0}-h\right)^{\gamma-1}-h^{\gamma-1}\right) \\
&+ \frac{-h^{\prime}\left(\varrho_{0}-h\right)\left(\left(\varrho_{0}-h\right)^{\gamma-1}-h^{\gamma-1}\right)-h\left(\varrho_{0}^{\prime}-h^{\prime}\right)\left(\left(\varrho_{0}-h\right)^{\gamma-1}-h^{\gamma-1}\right)}{\left(\varrho_{0}-h\right)^{\gamma}+h^{\gamma}} \\
&-(\gamma-1) \frac{h\left(\varrho_{0}-h\right)^{\gamma-1}\left(\varrho_{0}^{\prime}-h^{\prime}\right)-h^{\gamma-1}\left(\varrho_{0}-h\right) h^{\prime}}{\left(\varrho_{0}-h\right)^{\gamma}+h^{\gamma}}
\end{aligned}
$$

The first term on the RHS of (53) does not cause any difficulty: the condition (49) is sufficient to ensure the negativity of the term multiplying $h^{\prime}$. Here we are using (50). Considering the second and the third terms on the RHS we see that if

$$
\begin{aligned}
-\left(\varrho_{0}-2 h\right)\left(\left(\varrho_{0}-h\right)^{\gamma-1}-h^{\gamma-1}\right)+ & (\gamma-1) h\left(\varrho_{0}-h\right)^{\gamma-1} \\
& +(\gamma-1) h^{\gamma-1}\left(\varrho_{0}-h\right)<0
\end{aligned}
$$

for all $x \in \mathbb{T}$, then (51) is satisfied. It is easy to see that if $c_{\gamma}$ is sufficiently large (for example $c_{2} \geq 6 /(3-\sqrt{3})$ ), then (54) follows. This ends the proof. 
We may state the following result

Theorem 5.2. Let the initial data $F \in X_{\infty}$ be nonnegative functions with continuous second derivatives and such that (48), for some $\mu>0$, is satisfied. For any $T>0$ there exists $\varepsilon_{0}>0$ and $c_{\gamma}>0$ such that if

$$
\min _{x \in \mathbb{T}} F(k, x) \geq c_{\gamma} \max _{x \in \mathbb{T}} F(-k, x)
$$

is satisfied for given $k \in\{-1,1\}$, then for $\varepsilon \in\left[0, \varepsilon_{0}\right.$ [ the Cauchy Problem for Eq. (45), with the initial datum $F$, has a mild solution $f=f(t)$ in $X_{\infty}$ on $[0, T]$. Moreover,

$$
\sup _{t \in[0, T]}\left\|f(t)-\varrho(t)-\mathbf{h}\left(\frac{t}{\varepsilon}\right)\right\|_{\infty} \leq c_{T} \varepsilon,
$$

where

$$
\begin{array}{ll}
\varrho(t, k, x) & =F(k, x-k t), \\
\varrho(t,-k, x) & =0, \\
\mathbf{h}\left(\frac{t}{\varepsilon}, k, x\right) & =-h\left(\frac{t}{\varepsilon}, x\right), \\
\mathbf{h}\left(\frac{t}{\varepsilon},-k, x\right) & =h\left(\frac{t}{\varepsilon}, x\right),
\end{array}
$$

$\mathbf{h}=\bar{h}\left(\frac{t}{\varepsilon}, j, x\right), j=k,-k$,

$$
\mathbf{h}(0, x)=F(-k, x) \quad \forall x \in \mathbb{T} .
$$

Proof. Let $T>0$ be fixed. The proof follows by the analysis of the system (47) that, by (46), is equivalent to Eq. (45). The methods of Theorem 3.1 may be used to show the existence and uniqueness of the mild solution $f=f(t)$ in $X_{\infty}$ for any fixed $\varepsilon>0$. The solution satisfies

- $f(t) \in X_{\infty}$ and $f(t) \geq 0$ for a.a. $t \in[0, T]$,

- $\mu e^{-\frac{T}{\varepsilon}} \leq f(t)$ for a.a. $t \in[0, T]$,

- $\|f(t)\|_{\infty} \leq\left\|f_{0}\right\|_{\infty} e^{\frac{T}{\varepsilon}}$ for a.a. $t \in[0, T]$.

We may rewrite Eq. (45) in the following form

$$
\varepsilon \partial_{t} f_{j}^{\sharp}=\left(\frac{\chi\left(f_{j}+f_{-j}>0\right) f_{-j} f_{j}}{f_{j}^{\gamma}+f_{-j}^{\gamma}}\left(f_{j}^{\gamma-1}-f_{-j}^{\gamma-1}\right)\right)^{\sharp}, \quad j=k,-k,
$$

where "\#" is given by (14). Under the assumption (48) the functions $f(t, k, x+k t)$ and $f(t,-k, x-k t)$ are increasing and decreasing functions of $t$ (for any fixed $x)$, respectively, and we have

$$
\begin{aligned}
& f(t, k, x) \geq F(k, x-k t) \geq \min _{y \in \mathbb{T}} F(k, y)> \\
>\quad & c_{\gamma} \max _{y \in \mathbb{T}} F(-k, y) \geq c_{\gamma} F(-k, x+k t) \geq c_{\gamma} f(t,-k, x),
\end{aligned}
$$


for any $t>0$ and any $x \in \mathbb{T}$.

Now we assume (46) and consider Eq. (47), taking in consideration that $\bar{\varrho}+\varepsilon u+\varepsilon v>0$, we obtain

$$
\begin{aligned}
\partial_{t} u+k \partial_{x} u=\frac{1}{\varepsilon^{2}} \partial_{\tau} h+\frac{1}{\varepsilon} k \partial_{x} h & \\
& +\frac{(h+\varepsilon v)(\bar{\varrho}-h+\varepsilon u)^{\gamma}-(\bar{\varrho}-h+\varepsilon u)(h+\varepsilon v)^{\gamma}}{\varepsilon^{2}\left((\bar{\varrho}-h+\varepsilon u)^{\gamma}+(h+\varepsilon v)^{\gamma}\right)} \\
\partial_{t} v-k \partial_{x} v=- & \frac{1}{\varepsilon^{2}} \partial_{\tau} h+\frac{1}{\varepsilon} k \partial_{x} h \\
& +\frac{(\bar{\varrho}-h+\varepsilon u)(h+\varepsilon v)^{\gamma}-(h+\varepsilon v)(\bar{\varrho}-h+\varepsilon u)^{\gamma}}{\varepsilon^{2}\left((\bar{\varrho}-h+\varepsilon u)^{\gamma}+(h+\varepsilon v)^{\gamma}\right)} .
\end{aligned}
$$

Therefore, using (43) and (13),

$$
\begin{aligned}
\partial_{t} u+k \partial_{x} u & = \\
\frac{1}{\varepsilon} k \partial_{x} h+ & \frac{1}{\varepsilon^{2}}\left(\frac{(\bar{\varrho}-h+\varepsilon u)^{\gamma}(\bar{\varrho}+\varepsilon u+\varepsilon v)}{(\bar{\varrho}-h+\varepsilon u)^{\gamma}+(h+\varepsilon v)^{\gamma}}-\frac{(\bar{\varrho}-h)^{\gamma} \bar{\varrho}}{(\bar{\varrho}-h)^{\gamma}+h^{\gamma}}\right) \\
-\frac{1}{\varepsilon} u & +\frac{1}{\varepsilon^{2}}\left(\frac{h(\bar{\varrho}-h)^{\gamma}-(\bar{\varrho}-h) h^{\gamma}}{(\bar{\varrho}-h)^{\gamma}+h^{\gamma}}-\frac{h\left(\varrho_{0}-h\right)^{\gamma}-\left(\varrho_{0}-h\right) h^{\gamma}}{\left(\bar{\varrho}_{0}-h\right)^{\gamma}+h^{\gamma}}\right), \\
\partial_{t} v-k \partial_{x} v & = \\
\frac{1}{\varepsilon} k \partial_{x} h & +\frac{1}{\varepsilon^{2}}\left(\frac{(h+\varepsilon v)^{\gamma}(\bar{\varrho}+\varepsilon u+\varepsilon v)}{(\bar{\varrho}-h+\varepsilon u)^{\gamma}+(h+\varepsilon v)^{\gamma}}-\frac{h^{\gamma} \bar{\varrho}}{(\bar{\varrho}-h)^{\gamma}+h^{\gamma}}\right) \\
-\frac{1}{\varepsilon} v & +\frac{1}{\varepsilon^{2}}\left(\frac{(\bar{\varrho}-h) h^{\gamma}-h(\bar{\varrho}-h)^{\gamma}}{(\bar{\varrho}-h)^{\gamma}+h^{\gamma}}-\frac{\left(\varrho_{0}-h\right) h^{\gamma}-h\left(\varrho_{0}-h\right)^{\gamma}}{\left(\varrho_{0}-h\right)^{\gamma}+h^{\gamma}}\right) .
\end{aligned}
$$

Assume now that $\varrho, h, u, v$ are given and consider the following functions

$$
\Xi_{1}(\varepsilon)=\frac{(\bar{\varrho}-h+\varepsilon u)^{\gamma}(\bar{\varrho}+\varepsilon u+\varepsilon v)}{(\bar{\varrho}-h+\varepsilon u)^{\gamma}+(h+\varepsilon v)^{\gamma}},
$$

and

$$
\Xi_{2}(\varepsilon)=\frac{(h+\varepsilon v)^{\gamma}(\bar{\varrho}+\varepsilon u+\varepsilon v)}{(\varrho-\varrho+\varepsilon u)^{\gamma}+(h+\varepsilon v)^{\gamma}},
$$

We have

$$
\Xi_{i}(\varepsilon)-\Xi_{i}(0)=\varepsilon \int_{0}^{1} \Xi_{i}^{\prime}(\theta \varepsilon) \mathrm{d} \theta, \quad i=1,2 .
$$

The derivatives $\Xi_{i}^{\prime}(\varepsilon), i=1,2$, may be written as the sum

$$
\Xi_{i}^{\prime}(\varepsilon)=\Gamma_{i, 1}(\varepsilon) u+\Gamma_{i, 2}(\varepsilon) v,
$$


where, denoting $\Theta=\bar{\varrho}-h+\varepsilon u$ and $\Lambda=h+\varepsilon v$,

$$
\begin{aligned}
& \Gamma_{1,1}(\varepsilon)=\frac{\Theta^{2 \gamma}+\gamma \Theta^{\gamma-1} \Lambda^{\gamma}(\bar{\varrho}+\varepsilon u+\varepsilon v)+\Theta^{\gamma} \Lambda^{\gamma}}{\left(\Theta^{\gamma}+\Lambda^{\gamma}\right)^{2}}, \\
& \Gamma_{1,2}(\varepsilon)=\frac{\Theta^{2 \gamma}-\gamma \Theta^{\gamma} \Lambda^{\gamma-1}(\bar{\varrho}+\varepsilon u+\varepsilon v)+\Theta^{\gamma} \Lambda^{\gamma}}{\left(\Theta^{\gamma}+\Lambda^{\gamma}\right)^{2}}, \\
& \Gamma_{2,1}(\varepsilon)=\frac{\Theta^{\gamma} \Lambda^{\gamma}-\gamma \Theta^{\gamma-1} \Lambda^{\gamma}(\bar{\varrho}+\varepsilon u+\varepsilon v)+\Lambda^{2 \gamma}}{\left(\Theta^{\gamma}+\Lambda^{\gamma}\right)^{2}}, \\
& \Gamma_{2,2}(\varepsilon)=\frac{\Theta^{\gamma} \Lambda^{\gamma}+\gamma \Theta^{\gamma} \Lambda^{\gamma-1}(\bar{\varrho}+\varepsilon u+\varepsilon v)+\Lambda^{2 \gamma}}{\left(\Theta^{\gamma}+\Lambda^{\gamma}\right)^{2}} .
\end{aligned}
$$

Let us consider

$$
\begin{aligned}
\Gamma_{1,1}(\varepsilon) & =\frac{1+\frac{\Lambda^{\gamma}}{\Theta^{\gamma}} \frac{\varrho+\varepsilon u+\varepsilon v}{\Theta}+\frac{\Lambda^{\gamma}}{\Theta^{\gamma}}}{\left(1+\frac{\Lambda^{\gamma}}{\Theta \gamma}\right)^{2}} \\
& =\left(1+(h+\varepsilon v) \frac{\Lambda^{\gamma-1}}{\Theta^{\gamma}} \frac{\varrho+\varepsilon u+\varepsilon v}{\Theta}+\frac{\Lambda^{\gamma-1}}{\Theta^{\gamma}}\right)\left(1-\frac{\Lambda^{\gamma}}{\Theta^{\gamma}}+\ldots\right)^{2} .
\end{aligned}
$$

Now, by (48), (55) and (58) we get

$$
\begin{aligned}
& \bar{\varrho}-h+\varepsilon u \geq \min \left\{\bar{\varrho}-h, f_{k}\right\} \\
& \quad \geq \min \{F(k)-F(-k), F(k)\} \geq F(-k) \min \left\{c_{\gamma}-1, c_{\gamma}\right\} \geq \mu\left(c_{\gamma}-1\right),
\end{aligned}
$$

and

$$
\frac{\Lambda}{\Theta}=\frac{h+\varepsilon v}{\bar{\rho}-h+\varepsilon u} \leq \frac{\max \{F(-k), h\}}{\left(c_{\gamma}-1\right) F(-k)} \leq \frac{1}{c_{\gamma}-1} \rightarrow 0, \quad c_{\gamma} \rightarrow \infty .
$$

Performing analogous estimates for other $\Gamma_{i, j}$, we see that

$$
\begin{array}{ll}
\Gamma_{1,1}(\varepsilon)+\Gamma_{2,1}(\varepsilon)=1, & \\
\Gamma_{1,2}(\varepsilon)+\Gamma_{2,2}(\varepsilon)=1, & \\
\left|\Gamma_{1,1}(\varepsilon)\right| \leq 1+\sigma_{1,1}, & \left|\Gamma_{2,1}(\varepsilon)\right| \leq \sigma_{2,1}, \\
\left|\Gamma_{1,2}(\varepsilon)\right| \leq 1-\sigma_{1,2}, & \left|\Gamma_{2,2}(\varepsilon)\right| \leq \sigma_{2,2},
\end{array}
$$

where the positive constants $\sigma_{i, j}, i, j=1,2$, may be as small as we want if $c_{\gamma}$ is sufficiently large and also

$$
\begin{aligned}
& \left|\Gamma_{1,1}(\varepsilon)\right| \leq 1+\sigma_{1,1}^{\prime}(|h|+\varepsilon|v|), \\
& \left|\Gamma_{2,1}(\varepsilon)\right| \leq \sigma_{2,1}^{\prime}(|h|+\varepsilon|v|), \\
& \left|\Gamma_{2,2}(\varepsilon)\right| \leq \sigma_{2,2}^{\prime}(|h|+\varepsilon|v|),
\end{aligned}
$$


where again the positive constants $\sigma_{1,1}^{\prime}, \sigma_{2,1}^{\prime}, \sigma_{2,2}^{\prime}$ may be chosen as small as we want by taking sufficiently large $c_{\gamma}$.

In order to estimate the last term of Eq. (60) we assume that $\tau$ is fixed and consider

$$
\Psi(t)=\frac{(\varrho(t)-h(\tau)) h^{\gamma}(\tau)-h(\tau)(\varrho(t)-h(\tau))^{\gamma}}{\left.(\varrho(t)-h(\tau))^{\gamma}+h^{\gamma}(\tau)\right)} .
$$

By (13),

$$
\Psi(t)=\frac{h^{\gamma} \bar{\varrho}(t)}{(\varrho(t)-h)^{\gamma}+h^{\gamma}}-h,
$$

so that

$$
\Psi(t)-\Psi(0)=\frac{h^{\gamma} \bar{\varrho}(t)}{(\bar{\varrho}(t)-h)^{\gamma}+h^{\gamma}}-\frac{h^{\gamma} \varrho_{0}}{\left(\varrho_{0}-h\right)^{\gamma}+h^{\gamma}},
$$

and thus, using differentiability of the initial conditions, (58) and (61),

$$
\begin{aligned}
|\Psi(t)-\Psi(0)| & =t h^{\gamma}\left|\rho_{0}^{\prime}(\theta)\right|\left|\frac{(\bar{\varrho}(\theta)-h)^{\gamma}+h^{\gamma}-\gamma \bar{\varrho}(\theta)(\bar{\varrho}(\theta)-h)^{\gamma-1}}{\left((\bar{\varrho}(\theta)-h)^{\gamma}+h^{\gamma}\right)^{2}}\right| \\
& \leq t h^{\gamma}\left\|F^{\prime}\right\|_{\infty} \frac{(2+\gamma)\|F\|_{\infty}^{\gamma}}{\mu^{2}\left(c_{\gamma}-1\right)^{2}}=C_{\gamma} t h^{\gamma} .
\end{aligned}
$$

Then we have

$$
\begin{aligned}
\partial_{t} u & +k \partial_{x} u+\frac{1}{\varepsilon} u \\
& =\frac{1}{\varepsilon} k \partial_{x} h+\frac{u}{\varepsilon} \int_{0}^{1} \Gamma_{1,1}(\theta \varepsilon) \mathrm{d} \theta+\frac{v}{\varepsilon} \int_{0}^{1} \Gamma_{1,2}(\theta \varepsilon) \mathrm{d} \theta+\frac{t}{\varepsilon^{2}} \int_{0}^{1} \Psi^{\prime}(\theta t) \mathrm{d} \theta, \\
\partial_{t} v & -k \partial_{x} v+\frac{1}{\varepsilon} v \\
& =\frac{1}{\varepsilon} k \partial_{x} h+\frac{u}{\varepsilon} \int_{0}^{1} \Gamma_{2,1}(\theta \varepsilon) \mathrm{d} \theta+\frac{v}{\varepsilon} \int_{0}^{1} \Gamma_{2,2}\left(\theta_{2} \varepsilon\right) \mathrm{d} \theta-\frac{t}{\varepsilon^{2}} \int_{0}^{1} \Psi^{\prime}(\theta t) \mathrm{d} \theta .
\end{aligned}
$$

Integrating (66) along characteristics and using (63) and (64), we obtain

$$
\begin{aligned}
\|u(t)\|_{\infty} \leq \frac{C}{\varepsilon} \int_{0}^{t}\left(1+\frac{s}{\varepsilon}\right) e^{-\frac{s}{\varepsilon} \bar{\delta}} \mathrm{d} s+\frac{\sigma_{1,1}^{\prime}}{\varepsilon} \int_{0}^{t} e^{-\frac{s}{\varepsilon} \delta}\|u(s)\|_{\infty} \mathrm{d} s \\
+\sigma_{1,1}^{\prime} \int_{0}^{t}\|u(s)\|_{\infty}\|v(s)\|_{\infty} \mathrm{d} s+\frac{1-\sigma_{1,2}}{\varepsilon} \int_{0}^{t}\|v(s)\|_{\infty} \mathrm{d} s,(67) \\
\|v(t)\|_{\infty} \leq \frac{C}{\varepsilon} \int_{0}^{t} e^{-\frac{t-s}{\varepsilon}}\left(1+\frac{s}{\varepsilon}\right) e^{-\frac{s}{\varepsilon} \bar{\delta}} \mathrm{d} s \\
+\frac{\sigma_{2,1}}{\varepsilon} \int_{0}^{t} e^{-\frac{t-s}{\varepsilon}}\|u(s)\|_{\infty} \mathrm{d} s+\frac{\sigma_{2,2}}{\varepsilon} \int_{0}^{t} e^{-\frac{t-s}{\varepsilon}\|v(s)\|_{\infty} \mathrm{d} s,(68)}
\end{aligned}
$$


where $C$ denotes a generic constant independent of $\varepsilon$ and $\bar{\delta}<\min \left\{\gamma \delta, \delta_{1}\right\}>0$, see Lemma 5.1 and (65). For convenience of calculations, we fix $\bar{\delta}<1$.

We can re-write (68) as

$$
\|v(t)\|_{\infty} \leq C+\sigma_{2,1}\|u\|_{\infty, t}+\sigma_{2,2}\|v\|_{\infty, t}, \quad t \in[0, T],
$$

where $\|u\|_{\infty, t}=\sup _{s \in[0, t]}\|u(s)\|_{\infty}$. Taking $c_{\gamma}$ large enough for $\sigma_{2,2}$ to be smaller then 1 , we obtain

$$
\|v\|_{\infty, t} \leq C\left(1+\sigma_{2,1}\right)\|u\|_{\infty, t}, \quad t \in[0, T] .
$$

We may now refine the estimate (68), using (64) and (50), with $\delta$ replaced by $\bar{\delta}$,

$$
\begin{aligned}
\|v(t)\|_{\infty} \leq & \frac{C}{\varepsilon} \int_{0}^{t} e^{-\frac{t-s}{\varepsilon}}\left(1+\frac{s}{\varepsilon}\right) e^{-\frac{s}{\varepsilon} \bar{\delta}} \mathrm{d} s \\
& +\frac{\sigma_{2,1}^{\prime}}{\varepsilon} \int_{0}^{t} e^{-\frac{t-s}{\varepsilon}} e^{-\frac{s}{\varepsilon} \bar{\delta}}\|u(s)\|_{\infty} \mathrm{d} s+\frac{\sigma_{2,2}^{\prime}}{\varepsilon} \int_{0}^{t} e^{-\frac{t-s}{\varepsilon}} e^{-\frac{s}{\varepsilon} \bar{\delta}}\|v(s)\|_{\infty} \mathrm{d} s \\
& +\sigma_{2,1}^{\prime} \int_{0}^{t} e^{-\frac{t-s}{\varepsilon}}\|u(s)\|_{\infty}\|v(s)\|_{\infty} \mathrm{d} s+\sigma_{2,2}^{\prime} \int_{0}^{t} e^{-\frac{t-s}{\varepsilon}}\|v(s)\|_{\infty}^{2} \mathrm{~d} s .
\end{aligned}
$$

Therefore we find

$$
\begin{aligned}
\frac{1}{\varepsilon} \int_{0}^{t}\|v(s)\|_{\infty} \mathrm{d} s \leq & C+\sigma_{2,1}^{\prime}\|u\|_{\infty, t}+\sigma_{2,2}^{\prime}\|v\|_{\infty, t} \\
& +\sigma_{2,1}^{\prime}(T+\varepsilon)\|u\|_{\infty, t}\|v\|_{\infty, t}+\sigma_{2,2}^{\prime}(T+\varepsilon)\|v\|_{\infty, t}^{2} .
\end{aligned}
$$

By (67), (70) and (72) we obtain

$$
\begin{aligned}
\|u(t)\|_{\infty} \leq & C+\frac{\sigma_{1,1}^{\prime}}{\bar{\delta}}\|u\|_{\infty, t}+\sigma_{1,1}^{\prime} T C\|u\|_{\infty, t}\left(1+\sigma_{2,1}\|u\|_{\infty, t}\right) \\
& +\left(1-\sigma_{1,2}\right)\left(C+\sigma_{2,1}^{\prime}\|u\|_{\infty, t}+C \sigma_{2,2}^{\prime}\left(1+\sigma_{2,1}\|u\|_{\infty, t}\right)\right. \\
& +\sigma_{2,1}^{\prime}(T+\varepsilon) C\|u\|_{\infty, t}\left(1+\sigma_{2,1}\|u\|_{\infty, t}\right) \\
& \left.+\sigma_{2,2}^{\prime}(T+\varepsilon) C^{2}\left(1+\sigma_{2,1}\|u\|_{\infty, t}\right)^{2}\right)
\end{aligned}
$$

so that

$$
\|u\|_{\infty, t} \leq C+\sigma^{(1)}\|u\|_{\infty, t}+\sigma^{(2)}\|u\|_{\infty, t}^{2} .
$$

for any $t \in[0, T]$, where $\sigma^{(1)}$ and $\sigma^{(2)}$ are given by

$$
\begin{array}{r}
\sigma^{(1)}=\frac{\sigma_{1,1}^{\prime}}{\bar{\delta}}+\sigma_{1,1}^{\prime} T C+\left(1-\sigma_{1,2}\right)\left(\sigma_{2,1}^{\prime}+C \sigma_{2,1} \sigma_{2,2}^{\prime}\right) \\
+C\left(T+\varepsilon_{0}\right)\left(\sigma_{2,1}^{\prime}+2 C \sigma_{2,2}^{\prime} \sigma_{2,1}\right), \\
\sigma^{(2)=} \sigma_{1,1}^{\prime} \sigma_{2,1} T C+\left(\sigma_{2,1}^{\prime} \sigma_{2,1}+C \sigma_{2,2}^{\prime} \sigma_{2,1}^{2}\right) C\left(T+\varepsilon_{0}\right) .
\end{array}
$$


and tend to 0 with $\sigma_{i, j} \rightarrow 0$ and $\sigma_{i, j}^{\prime} \rightarrow 0$ (that is, for $c_{\gamma} \rightarrow \infty$ ). Thanks to this we may choose $c_{\gamma}$ large enough for $\sigma^{(1)}<1$ to obtain

$$
\|u\|_{\infty, t} \leq C\left(1+\sigma^{(2)}\|u\|_{\infty, t}^{2}\right),
$$

for any $t \in[0, T]$, where $C$ is a constant.

Let $y:=\|u\|_{\infty, t}, \alpha:=C$ and $\beta:=C \sigma^{(2)}$. Then, by (74), we have

$$
0 \leq-y+\alpha+\beta y^{2} \text {. }
$$

Considering the function $h(y)=\alpha-y+\beta y^{2}$, we may choose $\alpha>0$ and $\beta>0\left(\alpha \beta<\frac{1}{4}\right)$ such that

$$
h\left(y_{\min }\right)<0, \quad \text { for } \quad y_{\min }=\frac{1}{2 \beta} .
$$

Moreover $\left.y\right|_{t=0}=0$ and $y$ cannot experience jumps. Therefore, in particular, we have

$$
y \leq \frac{1}{2 \beta},
$$

and $\|u\|_{\infty, T}$ is bounded. Then (70) ends the proof.

\section{Acknowledgments}

M.L. acknowledges a financial support from the Polish Ministry of Science and Higher Education under the grant No. N N201 362536. J. B. acknowledges partial support from No. N N201 362536, partial support from the National Scientific Centre of Poland under the grant No. N N201 021133 and partial support from NRF Grant FA2007030300001.

\section{References}

[1] M. Agueh, R. Illner, A. Richardson, Analysis and simulations of a refined flocking and swarming model of Cucker-Smale type, Kinet. Relat. Models 4 (2011), 1-16.

[2] W. Alt, A. Deutsch, G. Dunn, Eds., Dynamics of Cell and Tissue Motion, Birkhäuser, 1997.

[3] L. Arlotti, A. Deutsch, M. Lachowicz, On a discrete Boltzmanntype model of swarming, Math. Comput. Modelling. 41, 10, 2005, 1193-1201.

[4] H. Bussemaker, A. Deutsch, and E. Geigant, Mean-field analysis of a dynamical phase transition in a cellular automaton model for collective motion, Phys. Rev. Lett. 78, 1997, pp. 5018-5021. 
[5] E. Ben-Jacob, O. Shochet, A. Tenenbaum, A. Czirók, and T. Vicsek, Cooperative formation of chiral patterns during growth of bacterial colonies, Phys. Rev. Lett. 75 (15), 1995, 2899-2902.

[6] M. Burger, V. Capasso, D. Morale, On an aggregation model with long and short range interactions, Nonlinear Anal.: Real World Appl. 8, 3 (2007), 939-958.

[7] J. Banasiak, V. Capasso, M.A.J. Chaplain, M. Lachowicz, J. Miẹkisz, Multiscale Problems in the Life Sciences. From Microscopic to Macroscopic, Lecture Notes in Mathematics 1940, Springer 2008.

[8] J.A. Carrillo, J.A. Cañizo, J. Rosado, Collective Behavior of Animals: Swarming and Complex Patterns, Arbor Ciencia, Pensamiento y Cultura 186, 746 (2010), 1035-1049.

[9] J.A. Carrillo, M. Fornasier, J. Rosado, and G. Toscani, Asymptotic flocking dynamics for the kinetic Cucker-Smale model, SIAM J. Math. Anal. 42, 2010, 218-236.

[10] P. Degond, S. Motsch, A macroscopic model for a system of swarming agents using curvature control, J. Statist. Phys. 143 (2011), 685-714.

[11] A. Deutsch, S. Dormann, Cellular Automaton. Modeling of Biological Pattern Formation, Birkhäuser, Boston 2005.

[12] E. Geigant, K. Ladishansky, A. Mogilner, An integro-differential model for orientational distribution of F-actin in cells, SIAM J. Appl. Math. 59, 1999, pp. 787-809.

[13] F. Golse, F. Salvarani, The nonlinear diffusion limit for generalized Carleman models: the initial-boundary value problem, Nonlinearity 20, 2007, 927-942.

[14] R. Erban, J. Haškovec, From individual to collective behaviour of coupled velocity jump processes: a locust example, to appear.

[15] M. Lachowicz, Asymptotic analysis of nonlinear kinetic equations: The hydrodynamic limit, in Lecture Notes on the Mathematical Theory of the Boltzmann Equation, World Sci., Singapore 1995, 65-148.

[16] M. Lachowicz, Individually-based Markov processes modeling nonlinear systems in mathematical biology, Nonlinear Analysis Real World Appl., 12 (4), 2011, 2396-2407.

[17] R. deLaubenfels, Existence Families, Functional Calculi and Evolution Equation, Springer, Berlin.

[18] F. Lutscher, Modeling alignment and movement of animals and cells, J. Math. Biol. 45, 2002, pp. 234-260. 
[19] R. Mach, F. Schweitzer, Modeling vortex swarming in Daphnia, Bull. Math. Biol. 69, 2007, 539-562.

[20] J. R. Mika, J. Banasiak, Singularly Perturbed Evolution Equations with Applications to Kinetic Theory, Series on Advances in Mathematics for Applied Sciences, vol. 34, World Scientific, Singapore, 1995.

[21] A. Mogilner, L. Edelstein-Keshet, Selecting a common direction. I. How orientational order can arise from simple contact responses between interacting cells, J. Math. Biol. 33(6), 1995, 619-660.

[22] D. Morale, V. Capasso, K. Oelschläger, An interacting particle system modelling aggregation behaviour: from individuals to populations, J. Math. Biol. 50 (2005) 49-66

[23] A. Palczewski, Exact and Chapman-Enskog Solutions for the Carleman Model, Math. Methods Appl. Sci. 6, 1984, 417-432.

[24] I. Primi, A. Stevens, J.J.L. Velázquez, Mass-selection in alignment models with non-deterministic effects, Comm. Partial Differential Eqs. 34 (2009), 419-456.

[25] F. Salvarani, G. Toscani, The diffusion limit of Carleman-type models in the range of very fast diffusion equation, J. Evol. Eqs. 9, 2009, $67-80$.

[26] F. Salvarani, J.L. Vázquez, The diffusive limit for Carleman-type kinetic model, Nonlinearity 18, 2005, 1223-1248.

[27] A.N. Tikhonov, A.B. Vasileva, A.G. Sveshnikov, Differential Equations. Nauka, Moscow (1985), in Russian.

[28] J. Toner, Y. Tu, Long-range order in a two-dimensional dynamical XY model: how birds fly together, Phys. Rev. Lett. 75(23), 1995, 4326-4329.

[29] Y. Wu, Y. Jiang, D. Kaiser, M. Alber, Social interaction in Myxobacterial swarming, PLOS Comput. Biol. 3, 12, 2007, 2546-2558.

[30] T.I. Zohdi, Mechanistic modeling of swarms, Comput. Methods Appl. Mech. Engrg. 198, 2009, 2039-2051. 